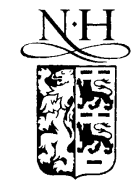

ELSEVIER
Available at

www.ComputerScienceWeb.com

POWERED SCIENCE (d) DIRECT

Pattern Recognition Letters 24 (2003) 1787-1794
Pattern Recognition Letters

www.elsevier.com/locate/patrec

\title{
Suppression of sampling moire in color printing by spline-based least-squares prefiltering
}

\author{
Dimitri Van De Ville ${ }^{a, *, 1}$, Wilfried Philips ${ }^{b}$, Ignace Lemahieu ${ }^{a}$, \\ Rik Van de Walle ${ }^{\text {a }}$ \\ a Department of Electronics and Information Systems (ELIS), Ghent University, \\ Sint-Pietersnieuwstraat 41, B-9000 Gent, Belgium \\ ${ }^{\mathrm{b}}$ Department of Telecommunications and Information Processing (TELIN), Ghent University, \\ Sint-Pietersnieuwstraat 41, B-9000 Gent, Belgium
}

\begin{abstract}
Many image processing systems, including those for printing applications, need sampling conversions for the representation of an image from one lattice to another. For example in the case of printing, classical halftoning requires new sample values on the halftone lattice. Although often considered as a straightforward procedure, resampling can cause so-called sampling moire due to aliasing. These artifacts are often very noticeable and as such undesirable, in particular for high-quality printing. In color printing, each color separation uses its own halftone lattice. Therefore, moire patterns will not only display an unexpected new frequency and orientation, but also influence the color appearance itself. These artifacts are frequently encountered in commercial (even high-quality) printing since the interpolation algorithms used in RIPs are simple (e.g., bilinear interpolation) and do not take into account the nature of the target lattice. Approaches such as simple low-pass filtering unacceptably blur the edges, while manual selective smoothing by an operator is very time-consuming.

This paper proposes an optimal prefilter which is based on the least-squares linear resampling paradigm. Our approach requires proper discrete/continuous models, i.e., for both the source and the target lattices, and computes the associated reconstruction function which minimizes the error between the representations in the continuous domain. The reconstruction function jointly takes into account the Nyquist areas of every color separation using a novel hexagonal spline model resulting into an optimal prefilter before halftoning. Experimental results show that after prefiltering, the images are much less prone to moire while not suffering from noticeable edge blurring.
\end{abstract}

(c) 2002 Elsevier Science B.V. All rights reserved.

Keywords: Color printing; Resampling; Sampling moire; Least-squares approximation; Bivariate splines; Hexagonal lattices

\footnotetext{
${ }^{*}$ Corresponding author. Tel.: +32-9-2648917; fax: +32-92643594.

E-mail addresses: dimitri.vandeville@epfl.ch, dimitri. vandeville@rug.ac.be (D. Van De Ville).

URL: http://www.elis.rug.ac.be/ dvdevill.

${ }^{1}$ Dimitri Van De Ville is a Research Assistant with the FWO Flanders, Belgium.
}

\section{Introduction}

Printing techniques are based upon a common principle. Since they can only put ink or not (i.e., a binary process), they rely on the limited spatial resolution of the human visual system (HVS) to 
create the perception of an intermediate shade of the ink's color. Halftoning techniques distribute small bi-level features on the paper. Ulichney (1987) gives an excellent overview of halftoning techniques in general. In this paper, we focus on classical halftoning or amplitude modulation, which places dots of varying sizes upon a regular lattice. This technique is still frequently used and very robust against ink-spreading problems.

Color printing technology is using another important property of the HVS which allows to synthesize (almost) any color by a proper combination of three primary ink colors: cyan (C), magenta (M), and yellow (Y). Black ink is also added for technical and economical reasons (for a detailed discussion of color halftoning we refer to Kang (1999)). Every color separation uses its own halftone lattice, but they are mutually rotated. Interaction of these periodic structures could easily give rise to moire-patterns known as interseparation moire. A common approach to minimize interseparation moire is by maximizing the angles of rotation. Typically, the black separation is at $45^{\circ}$, cyan at $105^{\circ}$, magenta at $75^{\circ}$, and yellow (which is the least visible) at $90^{\circ}$. If the separations are correctly aligned, an acceptable rosette structure becomes visible. An in-depth treatment of techniques to obtain moire-free separations can be found in literature (e.g., see Amidror, 2000).

Unfortunately, interseparation moire is not the only way moire patterns arise in color printing. Since the original image is resampled for each color separation to the corresponding lattice, aliasing of the image content can give rise to so-called sampling moire. As such, the moire patterns for each separation are different, showing each one a new low frequency at an "unnatural" orientation and creating a new color appearance when the separations are joined. Current resampling algorithms used in RIPs are fairly simple and do not incorporate any knowledge about the target lattice, e.g., nearest neighbour interpolation, bilinear interpolation, cubic convolution of Keys (1981). Therefore they do not prevent high-frequency components against turning into moire patterns. The advent of advanced scanners and digital cameras increases the availability of high-resolution images and likewise the possibility of high- frequency components being present. Typical "dangerous" image content includes fine textures, fabrics in clothes, and grills. One approach to prevent sampling moire is by applying low-pass filtering in order to suppress these high-frequency components. However, such a method unacceptably blurs edges. Another way is to let the operator manually smooth dangerous regions in the image, but such areas are difficult to predict and the job is time-consuming, as reported by Miller and Zaucha (1995) and Russ (1992). In the previous paper Van De Ville et al. (2000), we presented a non-linear resampling technique in order to obtain better results when resampling grayscale images by combining two linear resampling techniques. This method requires the computation of a risk image (estimating the amount of dangerous aliasing energy leading to moire patterns). Our current approach is less complex, it is applicable for color printing, and can be implemented as a prefilter which does not require to modify the RIP device.

This paper further elaborates the paper Van De Ville et al. (2002b). In order to obtain a joint optimization of the prefilter for every color separation, we introduce hexagonal splines. Those bivariate splines, although straightforward to construct by a simple recipe, appear novel and are wellsuited for our application. It is worthwhile to note that these splines are not equivalent to box splines (de Boor et al., 1993). Next, we apply the theory of least-squares resampling, as proposed by Unser et al. (1995), to our two-dimensional signal models. This will enable us to employ the proposed technique to design a proper prefilter which suppresses sampling moire in color printing. Finally, we draw conclusions and give some indications for future research.

\section{Generalized two-dimensional spline signal model}

A continuous/discrete model allows to construct a "smooth" signal in the continuous domain based on the sample values. Splines are a family of basis functions, which have a limited size of support, and expands as the order of the spline model increases. One of the most important spline families are the B-splines: piecewise polynomial func- 
tions, for a survey we refer to Unser et al. (1993). In general, they are not orthogonal, but they form a Riesz basis and satisfy the partition of unity condition. It is also interesting to mention the convolution property, which enables us to construct splines of the next order by convolving the spline with the first-order spline. Note that firstorder spline interpolation is better known as "nearest neighbour" interpolation; second-order spline interpolation as bilinear interpolation.

These models are appropriate for one-dimensional signals and can be easily extended to twodimensional rectangular lattices by means of the tensor-product. In this paper, we propose to construct a spline basis suitable for general periodic lattices, e.g., hexagonal lattices. A two-dimensional periodic lattice is characterized by the matrix $\boldsymbol{R}=\left[\boldsymbol{r}_{1} \mid \boldsymbol{r}_{2}\right]$ composed of the lattice vectors $\boldsymbol{r}_{1}$ and $\boldsymbol{r}_{2}$ which are linearly independent. A welldefined unique tiling cell is the Voronoi cell containing all points closer to its lattice site than to any other site. The indicator function for the Voronoi cell of the origin is easy to define:

$\chi_{\boldsymbol{R}}(\boldsymbol{x})= \begin{cases}1 & \boldsymbol{x} \in \text { Voronoi cell, } \\ 1 / m_{\boldsymbol{x}} & \boldsymbol{x} \text { edge Voronoi cell, } \\ 0 & \boldsymbol{x} \notin \text { Voronoi cell, }\end{cases}$

where $m_{x}$ is the number of lattice points to which $\boldsymbol{x}$ is equidistant. Note that $\chi_{\boldsymbol{R}}(\boldsymbol{x})$ tiles the plane by definition. Now we choose the first-order spline to be $\beta^{0}(\boldsymbol{x})=\chi_{\boldsymbol{R}}(\boldsymbol{x})$. Spline functions of higher order are constructed by subsequent convolutions:

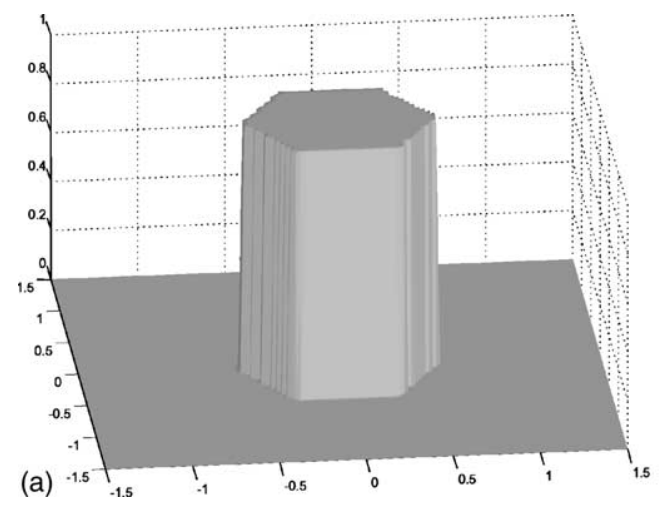

$\beta^{n}(\boldsymbol{x})=\frac{\beta^{0} \otimes \beta^{n-1}(\boldsymbol{x})}{|\operatorname{det}(\boldsymbol{R})|}, \quad n \geqslant 1$,

where each spline function is normalized by the surface area of the Voronoi cell $|\operatorname{det}(\boldsymbol{R})|$. The signal space $S\left(\beta^{n}\right)$ based on the shifted splines $\beta^{n}$ contains the signal models $s(\boldsymbol{x})$ :

$$
\begin{gathered}
S\left(\beta^{n}\right)=\left\{s(\boldsymbol{x}) \mid s(\boldsymbol{x})=\sum_{\boldsymbol{k} \in \mathbb{Z}^{2}} c(\boldsymbol{k}) \beta^{n}(\boldsymbol{x}-\boldsymbol{R} \boldsymbol{k}) ;\right. \\
c(\boldsymbol{k}) \in \mathbb{R}\} .
\end{gathered}
$$

In order to represent a function $g(\boldsymbol{x})$, the spline coefficients $c(\boldsymbol{k})$ must make $s(\boldsymbol{R} \boldsymbol{k})=g(\boldsymbol{R} \boldsymbol{k})$. For the first- and second-order this condition is easy to satisfy by choosing $c(\boldsymbol{k})=g(\boldsymbol{R} \boldsymbol{k})$, while higher orders need an inverse filter operation to obtain the right values for $c(\boldsymbol{k})$. For this paper we only consider first- and second-order spline models. Nevertheless, the generalization of the proposed technique to higher orders is straightforward. We introduce the notation of the spline with a subscript $\boldsymbol{R}$ to refer to a sampled version on the lattice $\boldsymbol{R}$ :

$\beta_{\boldsymbol{R}}^{n}(\boldsymbol{x})=\beta^{n}(\boldsymbol{x}) \sum_{\boldsymbol{k}} \delta(\boldsymbol{x}-\boldsymbol{R} \boldsymbol{k})$.

As an illustration, consider the regular hexagonal lattice, characterized by the matrix

$\boldsymbol{R}=\left[\begin{array}{cc}\sqrt{3} / 2 & 0 \\ -1 / 2 & 1\end{array}\right]$

Fig. 1. The generalized spline functions for a hexagonal lattice. (a) First order; (b) second order. 
We first define the first-order hexagonal spline $(n=0)$ as the indicator function of the Voronoi cell of the lattice. Fig. 1(a) shows the first-order hexagonal spline. Note that it fills up the twodimensional space if it is copied upon each lattice site (i.e., the partition of unity condition is fulfilled). A convolution of this spline with itself (and the proper normalization by its surface area) results into the second-order spline $(n=1)$, shown in Fig. 1(b). Using this recipe, splines of arbitrary order can be constructed, becoming smoother as the order increases. We have proven that this spline family fulfills the necessary conditions (Riesz basis, convexity, positivity) to be a sensible continuous/discrete model. Additionally, the order of approximation corresponds to the nomenclature we introduced, i.e., $(n+1)$ th order. An analytical expression of the Fourier transform has been obtained. For more in-depth treatment of these generalized splines, we refer to Van De Ville et al. (2002a).

\section{Least-squares based prefiltering}

Artifacts in color printing caused by sampling moire are very undesirable, not only because they introduce new frequency components (i.e., with a new frequency and a new orientation), but also because new color tints due to different aliasing for each color separation arise. Since the Nyquist areas of the lattices of each color separation are different (i.e., mutually rotated), moire patterns are different in each color separation and might interfere with each other. This phenomenon will be illustrated later in the following section.
The first step for the derivation of a prefilter is to select the discrete/continuous signal models to represent the sampled images on their source and target lattice. An interesting approach is leastsquares resampling: the reconstruction function minimizes the squared error between the continuous model for the image on the source lattice and the model for the image on the target lattice using the new sample values, see also Unser et al. (1995) and Unser (2000). Consider a source lattice $\boldsymbol{R}$ and a target lattice $\widetilde{\boldsymbol{R}}$, where the notation with the tilde refers to the target lattice. Based on the twodimensional splines of the preceding section, we can easily reconstruct an image in the continuous domain, using a reconstruction of the approximation spaces $S\left(\beta^{n}\right)$ on the one side and $S\left(\tilde{\beta}^{n}\right)$ on the other side. We are aiming at a convolutionbased scheme

$\tilde{s}(\boldsymbol{R} \boldsymbol{k})=h(\boldsymbol{R} \boldsymbol{k}) ; \quad h(\boldsymbol{x})=\sum_{\boldsymbol{k}} \Phi^{n}(\boldsymbol{x}-\boldsymbol{R} \boldsymbol{k}) g(\boldsymbol{R} \boldsymbol{k})$,

where the reconstruction function $\Phi^{n}(\boldsymbol{x})$ realizes the least-squares approximation between both spline reconstructions, i.e., solving the minimization problem

$\min _{\tilde{s} \in S(\tilde{\beta})}\|s-\tilde{s}\|$.

Fig. 2 illustrates the principle of least-squares resampling by a block diagram. Since the error $s(\boldsymbol{x})-\tilde{s}(\boldsymbol{x})$ must be orthogonal to $S\left(\tilde{\beta}^{n}\right)$, we can write

$\left\langle s^{n}(\boldsymbol{x})-\tilde{s}^{n}(\boldsymbol{x}), \tilde{\beta}^{n}(\boldsymbol{x}-\widetilde{\boldsymbol{R}} \boldsymbol{k})\right\rangle=0$,

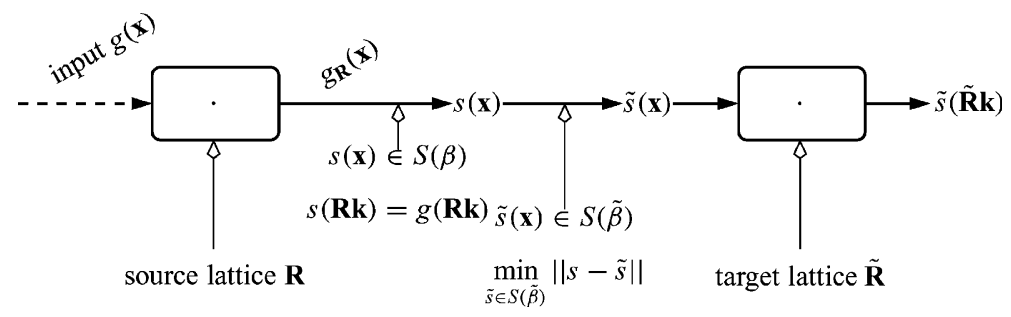

Fig. 2. Resampling by means of a least-squares approximation obtains new sample values $\tilde{\boldsymbol{s}}(\underset{\boldsymbol{R}}{\widetilde{R}})$ such that the error $\|s-\tilde{s}\|^{2}$ between the signal model $s(\boldsymbol{x})$ on the source lattice $\boldsymbol{R}$ and the signal model $\tilde{\boldsymbol{s}}(\boldsymbol{x})$ on the target lattice $\widetilde{\boldsymbol{R}}$ is minimal. 
which can be worked out as

$$
\begin{aligned}
& \left\langle s^{n}(\boldsymbol{x}), \tilde{\beta}^{n}(\boldsymbol{x}-\widetilde{\boldsymbol{R}} \boldsymbol{k})\right\rangle \\
& =\left\langle\sum_{\boldsymbol{l}} \tilde{c}(\boldsymbol{l}) \tilde{\beta}^{n}(\boldsymbol{x}-\widetilde{\boldsymbol{R}} \boldsymbol{l}), \tilde{\beta}^{n}(\boldsymbol{x}-\widetilde{\boldsymbol{R}} \boldsymbol{k})\right\rangle \\
& =\sum_{\boldsymbol{l}} \tilde{c}(\boldsymbol{l})\left\langle\tilde{\beta}^{n}(\boldsymbol{x}-\widetilde{\boldsymbol{R}} \boldsymbol{l}), \tilde{\beta}^{n}(\boldsymbol{x}-\widetilde{\boldsymbol{R}} \boldsymbol{k})\right\rangle \\
& =|\operatorname{det}(\widetilde{\boldsymbol{R}})|\left(\left[\sum_{l} \delta(\boldsymbol{x}-\widetilde{\boldsymbol{R}} \boldsymbol{l}) \tilde{c}(\boldsymbol{l})\right] \otimes \tilde{\beta}^{2 n+1}\right)(\widetilde{\boldsymbol{R}} \boldsymbol{k}) .
\end{aligned}
$$

Solving this equation for the coefficients $\tilde{c}(\boldsymbol{k})$ results into

$\tilde{c}(\boldsymbol{k})=\frac{s^{n} \otimes \tilde{\beta}^{n} \otimes\left(\tilde{\beta}_{\tilde{\boldsymbol{R}}}^{2 n+1}\right)^{-1}(\widetilde{\boldsymbol{R}} \boldsymbol{k})}{|\operatorname{det}(\widetilde{\boldsymbol{R}})|}$.

As such, the reconstruction function $\Phi^{n}(\boldsymbol{x})$ for the convolution-based scheme of Eq. (6) is

$$
\Phi^{n}(\boldsymbol{x})=\frac{\overbrace{\left(\beta_{\boldsymbol{R}}^{n}\right)^{-1} \otimes \beta^{n}}^{(1)} \otimes \overbrace{\tilde{\beta}^{n} \otimes\left(\tilde{\beta}_{\tilde{\boldsymbol{R}}}^{2 n+1}\right)^{-1}}^{(2)} \otimes \overbrace{\tilde{\beta}_{\tilde{\boldsymbol{R}}}^{n}}^{(3)}(\boldsymbol{x})}{|\operatorname{det}(\boldsymbol{R})|},
$$

where the overbraced expressions represent respectively: the spline transform of the spline model on the source lattice, the least-squares filter, and the reconstruction filter of the spline model on the target lattice.

For more details on the derivation and the computation of Eq. (10) we also refer to Van De Ville et al. (2002a). The first-order solution $\Phi^{0}(\boldsymbol{x})$ corresponds to the well-known principle of "surface projection": neighbouring samples of the source lattice are weighted by the relative overlap of source's and target's cell. No inverse filters are needed and the support is limited. For the second-order least-squares approximation, the reconstruction function contains an inverse filter $\left(\tilde{\beta}_{\tilde{\boldsymbol{R}}}^{3}\right)^{-1}$, which implicates that the theoretical support of $\Phi^{1}(\boldsymbol{x})$ is the whole plane. However, the fast decay makes an approximation on a limited support appropriate with negligible error.

\section{Application to color printing}

Instead of proposing different reconstruction functions based on the lattice of each color sepa-

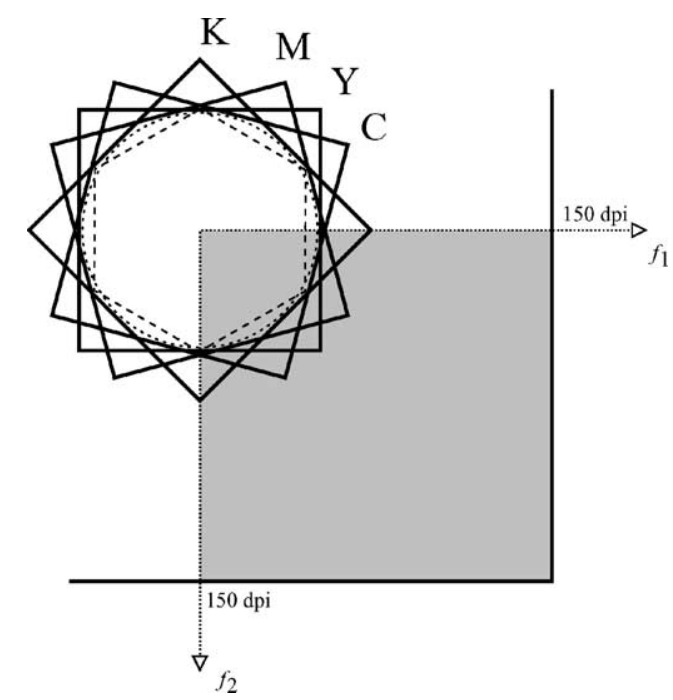

Fig. 3. The outer square is the Nyquist area of the source lattice, while the small rotated squares are the Nyquist areas of the color separations. An hexagonal cell is able to cover the largest enclosed circle most efficiently. The gray region in one quadrant corresponds to the frequency region represented by the test image zoneplate.

ration (in a least-squares sense), we propose a joint criterion. For the purpose of illustration, Fig. 3 shows the frequency domain and each of the Nyquist areas of the color separations and their largest enclosed circle. The resolution of the square source lattice is assumed to be $300 \times 300 \mathrm{dpi}$. The resolution of the halftone target lattices is $100 \times$ $100 \mathrm{dpi}$. Note the correspondence with the general rule of thumb that the source lattice should be of 2-3 times higher resolution than the halftone resolution. Next, we consider the enclosed circle of all Nyquist areas of the color separations. The lattice of which its Voronoi cell covers most efficiently the surface area of the enclosed circle is the hexagonal lattice (and so is its reciprocal cell in the frequency domain, see also Petersen and Middleton (1962), Mersereau (1979), Ulichney (1987)). We computed the least-squares reconstruction function for resampling from the source square lattice to this hexagonal target lattice, and used it as a prefilter before halftoning since the incorporation into PostScript or a RIP device is difficult to realize. The advantage of considering the color separations together, is obviously that we only need a 
single prefilter which can even be applied before the image is converted to the CMYK colorspace.

We first illustrate the origin of moire patterns by considering the test image "zoneplate" (a twodimensional frequency sweep). Fig. 4(a) shows the result of the zoneplate after regular halftoning. Severe moire patterns appear differently for each color separation; the combination also shows new colors.

Fig. 5 shows the optimal prefilters based on the least-squares approximation paradigm between the source lattice and the hexagonal target lattice. The filter coefficients are taken at the integer lattice sites and applied after normalization, i.e., $\sum_{k} \Phi^{n}(\boldsymbol{R} \boldsymbol{k})=1$. The numerical computation procedure of the reconstruction function is iteratively and can also be found in (Van De Ville et al., 2002a).

Fig. 6 shows the test image zoneplate after prefiltering and halftoning. The moire patterns are very well suppressed in both color separations. The influence of the order of the models used for original and resampled image is almost unnoticeable. In fact, higher orders $(n \geqslant 2)$ are not very desirable: (1) the size of the support for a decent approximation of the prefilter increases, (2) the frequency response gets sharper and ringing artifacts might appear. Of course, its important to
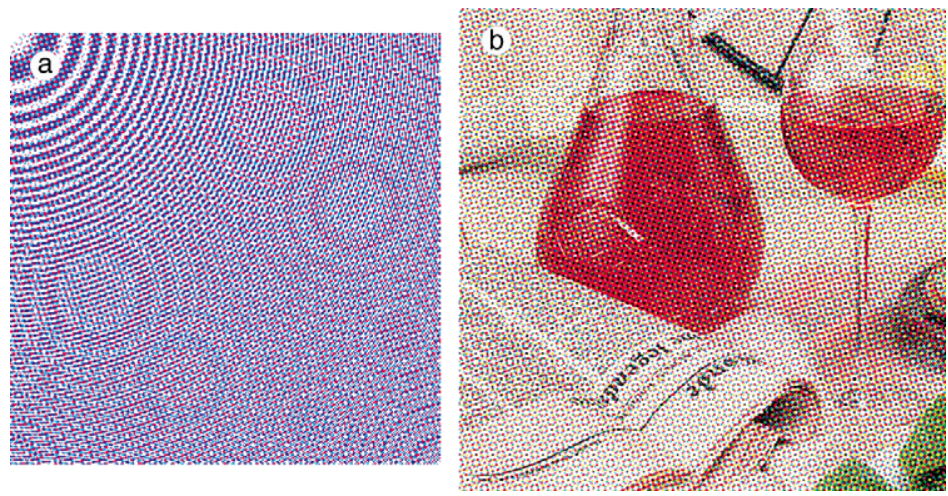

Fig. 4. (a) The test image zoneplate after regular halftoning. Note (different) moire patterns occur in the cyan and magenta separation. The digital annex contains the CMYK TIFF "zone_CM.tif” which allows the interested reader to view the individual separations. (b) A normal test image (part of the ISO 400 test set) after regular halftoning.
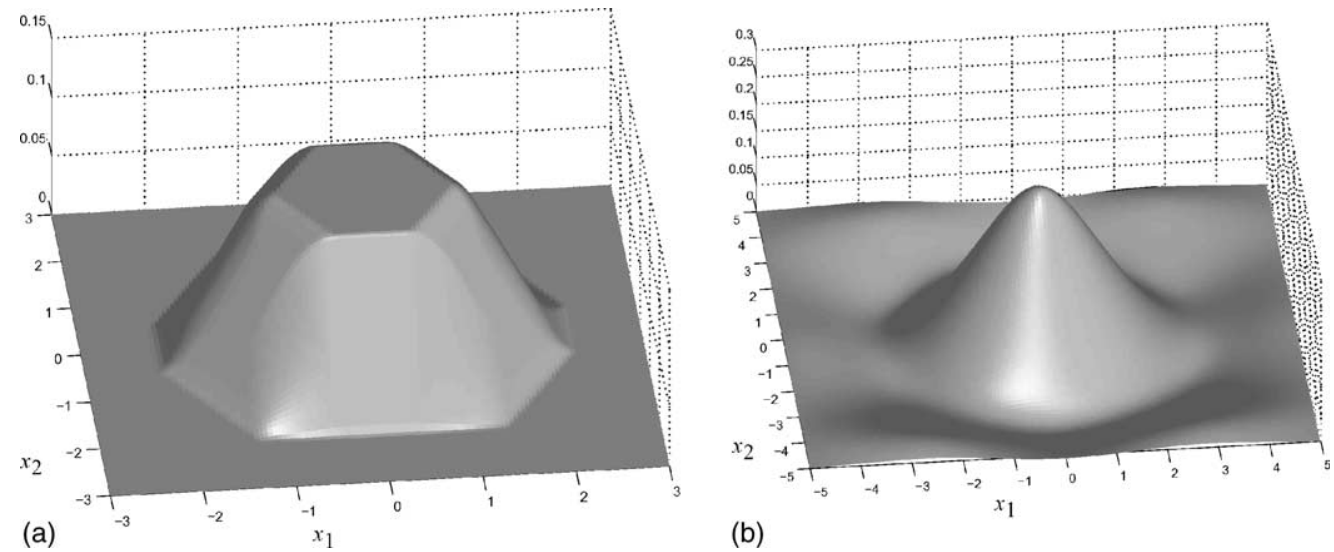

Fig. 5. The optimal prefilter based on the least-squares approximation between the source lattice and the hexagonal target lattice. The values at the integer lattice sites are used as filter coefficients after normalization. (a) First order; (b) second order. 

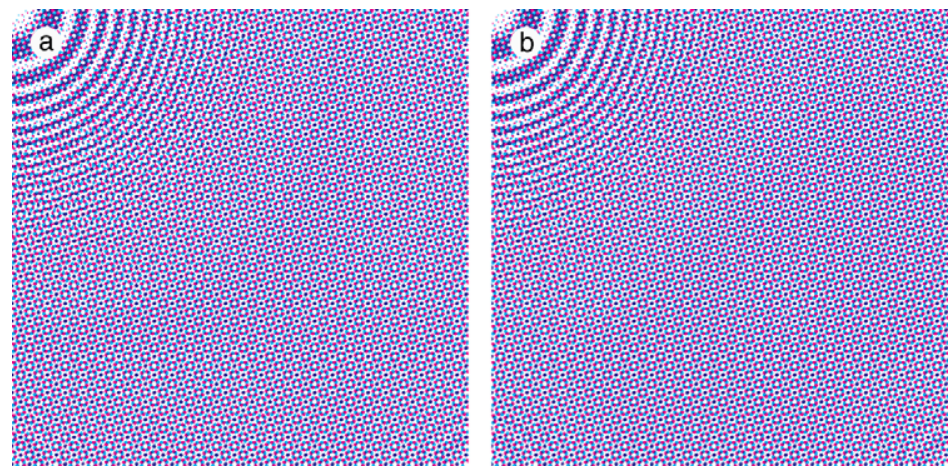

Fig. 6. The test image zoneplate (cyan and magenta) after regular halftoning, but after applying the least-squares prefilter. (a) First order; (b) second order.
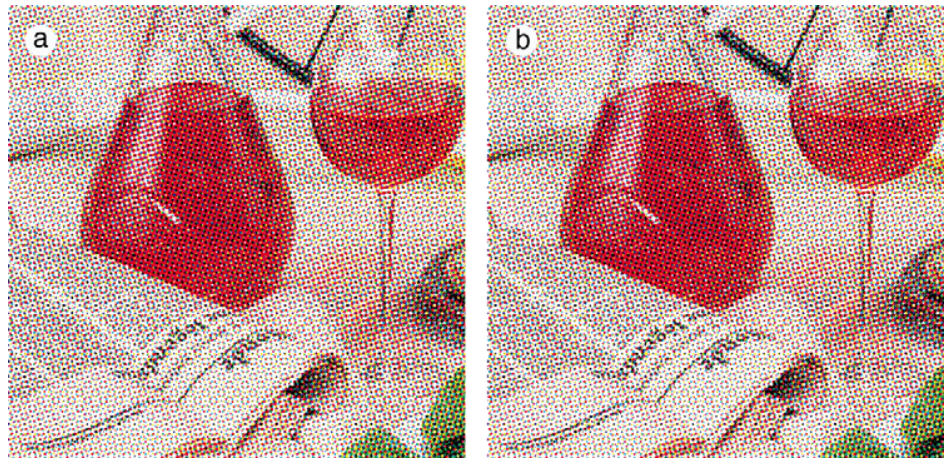

Fig. 7. Normal test image. (a) Halftoned after first-order least-squares prefiltering. (b) Halftoned after second-order least-squares prefiltering.

examine that the filter does not deteriorate normal images by blurring the edges. With the set of test images from ISO 12640:1997 (1997), we could not see any differences of the halftoned image with and without prefilter. Figs. 4(b) and 7 show a part of an ISO400 test image which exhibit no noticeable difference between the image after halftoning with and without prefiltering. The second-order should produce sharper results, but the halftoning process almost completely masks this effect.

\section{Conclusions}

Moire patterns in color printing are very unwanted artifacts which could ruin a print job. The advent of advanced scanners and digital cameras increases the availability of high-resolution images and even so the possibility of high-frequency components giving cause to moire patterns. Resampling techniques such as nearest neighbour and bilinear interpolation are common practice, but they do not incorporate the properties of the target lattice in any way. In the case of color printing, moire patterns due to aliasing can exhibit new frequencies, orientations, and color tints. Based on a novel class of two-dimensional spline models, we propose a reconstruction function based on a leastsquares approximation and apply it as a prefilter. The assumed target lattice is hexagonal in order to jointly optimize for all color separations. Results show that moire patterns are well suppressed while there is no visual loss of edge sharpness in "normal" images.

Future research could try to incorporate the algorithm into the resampling device itself, or 
precompensate for the "common" resampling technique it implements. Another interesting option is to choose the orders of the spline models differently. For example, the order of the spline model for the target lattice could be chosen equal to one (corresponding to a rough model for the halftoned image), while the order of the spline model for the source lattice could be chosen higher.

\section{References}

Amidror, I., 2000. The Theory of the Moiré Phenomenon. Computational Imaging and Vision, vol. 15. Kluwer Academic Publishers, Dordrecht.

de Boor, C., Höllig, K., Riemenschneider, S., 1993. Box splines. Applied Mathematical Sciences, vol. 98. Springer-Verlag.

ISO 12640:1997, 1997. CMYK standard colour image data. CD-ROM.

Kang, H.R., 1999. Digital Color Halftoning. SPIE Optical Engineering Press.

Keys, R.G., 1981. Cubic convolution interpolation for digital image processing. IEEE Trans. Acoust. Speech Signal Process. 29 (6), 1153-1160.

Mersereau, R., 1979. The processing of hexagonally sampled two-dimensional signals. Proc. IEEE 67 (6), 930-949.
Miller, M.D., Zaucha, R., 1995. The Color PC-Production Techniques. Hayden Books, New Jersey.

Petersen, D., Middleton, D., 1962. Sampling and reconstruction of wave-number-limited functions in N-dimensional Euclidean spaces. Inf. Control 5, 279-323.

Russ, J.C., 1992. The Image Processing Handbook. CRC Press, Boca Raton.

Ulichney, R.A., 1987. Digital Halftoning. MIT Press, Cambridge, MA.

Unser, M., 2000. Sampling-50 years after Shannon. Proc. IEEE 88 (4), 569-587.

Unser, M., Aldroubi, A., Eden, M., 1993. B-spline signal processing. IEEE Trans. Signal Process. 41 (2), 821848.

Unser, M., Aldroubi, A., Eden, M., 1995. Enlargement or reduction of digital images with minimum loss of information. IEEE Trans. Image Process. 4 (3), 247-258.

Van De Ville, D., Denecker, K., Philips, W., Lemahieu, I., 2000. Nonlinear resampling for edge preserving moiré suppression. J. Electron. Imaging 9 (4), 534-547.

Van De Ville, D., Philips, W., Lemahieu, I., 2002a. Leastsquares spline resampling to a hexagonal lattice. Signal Process. Image Commun. 17 (5), 393-408.

Van De Ville, D., Philips, W., Lemahieu, I., 2002b. Suppressing sampling moiré by least-squares prefiltering in color printing. In: Proceedings of the First European Conference on Color in Graphics, Image and Vision. IS \& T, Poitiers, France, pp. 100-103. 